\section{A CHECKLIST OF BIRDS OF THE NATIONAL INSTITUTE OF TECHNOLOGY CAMPUS, KOZHIKODE, KERALA}

\section{J. Praveen ${ }^{1}$ and Job K. Joseph ${ }^{2}$}

1 14/779(2), Ambadi, K. Medu P.O., Palakkad, Kerala 678013, India ${ }^{2}$ Thottacherry House, Vazhappally (W), Changanacherry, Kerala 686103, India

Email: ${ }^{1}$ painted_stork@yahoo.com, ${ }^{2}$ jobkjoseph@yahoo.com.

An article by Palot and Pramod (2000) provided a non-annotated checklist of birds found in Calicut University Campus, Kozhikode, Kerala. In this note, we provide a corresponding checklist with relative abundance indicators for a college campus lying east ( $25 \mathrm{~km}$ east) of the Calicut University Campus. A comparison among the checklists is attempted as both the campuses have similar sizes and habitats. The study period is also of comparable duration though during different years, 199396 for Palot and Pramod (2000) while 1996-2000 in this case.

Study area: The National Institute of Technology (formerly Regional Engineering College) campus in Kozhikode (formerly Calicut) is situated roughly $22 \mathrm{~km}$ north-east of Kozhikode city, almost at the foothills of the Western Ghats, with an approximate area of 120ha. The topography of the campus is undulating with laterite hillocks supporting mixed groves and bushes with some acacia/cashew plantations in the slopes. The vegetation is dominated by bushes like Calicopteris floribunda, Lantana camara, Rauvolfia tetraphylla, Eupatorium odoratum and Clerodendrum infortunatum interspersed with tress like Mangifera indica, Macaranga peltata, Alstonia scholaris, Azadirachta indica and Ficus benghalensis. The road that leads from Kozhikode to the town of Areekode bisects the campus. Most of the administrative and academic buildings are housed in the southeastern corner of the campus. The residential buildings on the northwestern side of the campus are well spaced with lush, wild patches of undergrowth sprayed with native tree species. This includes several fruit-bearing and berry-bearing species (like Mangifera indica \& Ficus spp.), deliberately planted by the authorities over the due course of time. Rest of the campus is uninhabited forest, almost fully contiguous, except for the presence of a couple of football fields cradled in the valley between two hillocks. The presence of these grounds provide better pedestrian access to these forested areas of the campus. A large section in the southern part of this forested zone comprises of a plantation of exotic Acacia trees, a best example of how not to add tree cover!

The authors visited all the habitats and trek routes in the campus during all months of the year with regular frequency during the study period. Hence, the checklist can be considered more or less comprehensive for the region.

Noteworthy species:

1. Malabar Whistling Thrush (Myophonus horsfieldii)

A handful of sightings in the forested patches during post- monsoon months. This might indicate a dispersal of this species from the breeding grounds during the nonbreeding season. The melodious song was never heard though the authors recorded the single noted "swing" whistle. Palot and Pramod (2000) had not reported this species from their study site possibly indicating that the dispersal does not reach that far from the Western Ghats.

\section{Indian Rufous Babbler (Turdoides subrufa)}

Very local. All sightings of this species were at a single locality within the campus and hence could probably be a remnant population. Not more than 10 individuals noted at a time. Not recorded by Palot and Pramod (2000).

\section{Brown-breasted Flycatcher (Muscicapa muttui)}

There was just one single sighting from the campus probably on passage. Not recorded by Palot and Pramod (2000).

\section{Yellow-wattled Lapwing (Vanellus malabaricus)}

This is one species that has been severely affected by the acquisition of open fallow land. This threat, though widespread throughout the country, can be much appreciated in Kerala as the remaining population of this species has localized itself to college campuses where open playgrounds abound. NIT campus has got a good population of these lapwings. Up to 30 -odd individuals have been recorded as part of a single group.

Threats and Conservation: Being an academic campus, the remaining wooded patches are always under threat from constant development for new infrastructure. The college nature club thwarted several tree-cutting plans of the authorities during the authors' stay at NIT Calicut. A plan was also chalked out to selectively replace the existing acacia plantations with natural tree cover and afforestation schemes with the help of Social Forestry division of the Forest Department. Saplings of indigenous trees were planted in several areas where acacia was removed. Luckily none of the playgrounds were converted to buildings during the period; any such attempts will severely threaten the existence of birds like Yellow-wattled Lapwings.

Table 1. Comparison of results between NIT Campus and Calicut University Campus.

\begin{tabular}{lll}
\hline Attribute & $\begin{array}{l}\text { University Campus } \\
\text { (Palot \& Pramod, 2000) }\end{array}$ & $\begin{array}{l}\text { NIT Campus } \\
\text { (This paper) }\end{array}$ \\
\hline Period & $\begin{array}{l}1993-1996 \\
200 \text { hectares }^{1}\end{array}$ & $\begin{array}{l}1996-2000 \\
120 \text { hectares }\end{array}$ \\
Area & 124 & 118 \\
Species & 14 & 19 \\
Migrants & $0^{2}$ & 4 \\
Local Migrants & $0^{3}$ & 1 \\
Vagrants & 8 & 6 \\
Primary Hole Nesting Species & & 3 \\
(Woodpeckers \& Barbets) & 4 & 4 \\
Nest Parasite Species (Cuckoos) & 1 & \\
Undergrowth Species & & 12 \\
(Pheasants, Quails \& Thrushes) & & $1^{5}$ \\
$\begin{array}{l}\text { Birds of Prey (Eagles, Falcons } \\
\text { \& Owls) }\end{array}$ & 13 & $1^{4}$ \\
Western Ghat Endemics & & \\
\hline
\end{tabular}

${ }^{1}$ A probable error in [3], where area is mentioned as $25 \mathrm{~km}^{2}$ which is unlikely

2 Palot and Pramod do not classify this status

${ }^{3}$ Palot and Pramod do not classify this status.

${ }^{4}$ Small Sunbird

${ }^{5}$ Rufous Babbler 
Table 2. Birds of National Institute of Technology Campus, Kozhikode

\begin{tabular}{|c|c|c|c|}
\hline S. No. & Common Name & Scientific Name & Status \\
\hline 1. & Little Cormorant & Phalacrocorax niger & C \\
\hline 2. & Little Egret & Egretta garzetta & $\mathrm{R}$ \\
\hline 3. & Median Egret & Mesophoyx intermedia & $\mathrm{R}$ \\
\hline 4. & Cattle Egret & Bubulcus ibis & $\mathrm{F}$ \\
\hline 5. & Large Egret & Casmerodius albus & $\mathrm{R}$ \\
\hline 6. & Indian Pond Heron & Ardeola grayii & C \\
\hline 7. & Black-crowned Night Heron & Nycticorax nycticorax & $\mathrm{R}$ \\
\hline 8. & Chestnut Bittern & Ixobrychus cinnamomeus & $\mathrm{R}$ \\
\hline 9. & Black-shouldered Kite & Elanus caeruleus & $\mathrm{R}$ \\
\hline 10. & Black Kite & Milvus migrans & $\mathrm{F}$ \\
\hline 11. & Brahminy Kite & Haliastur indus & $\mathrm{F}$ \\
\hline 12. & Shikra & Accipiter Badius & $\mathrm{F}$ \\
\hline 13. & Booted Eagle & Hieraaetus pennatus & $\mathrm{R}$ \\
\hline 14. & Changeable Hawk Eagle & Spizaetus cirrhatus & $\mathrm{R}$ \\
\hline 15. & Western Marsh Harrier & Circus aeruginosus & $\mathrm{R}, \mathrm{M}$ \\
\hline 16. & Crested Serpent Eagle & Spilornis cheela & $\mathrm{F}$ \\
\hline 17. & Common Kestrel & Falco tinnunculus & $\mathrm{R}$ \\
\hline 18. & Red Spurfowl & Galloperdix spadicea & $\mathrm{F}$ \\
\hline 19. & White-breasted Waterhen & Amaurornis phoenicurus & $\mathrm{F}$ \\
\hline 20. & Bronze-winged Jacana & Metopidius indicus & $\mathrm{R}$ \\
\hline 21. & Common Sandpiper & Tringa hypoleucos & R. M \\
\hline 22. & Green Sandpiper & Tringa ochropus & $\mathrm{R}, \mathrm{M}$ \\
\hline 23. & Little-ringed Plover & Charadrius dubius & $\mathrm{R}, \mathrm{M}$ \\
\hline 24. & Red-wattled Lapwing & Vanellus indicus & $\mathrm{C}$ \\
\hline 25. & Yellow-wattled Lapwing & Vanellus malabaricus & $\mathrm{C}$ \\
\hline 26. & Common Snipe(?) & Gallinago gallinago & $\mathrm{R}, \mathrm{M}$ \\
\hline 27. & Rock Pigeon & Columba livia & $\mathrm{C}$ \\
\hline 28. & Spotted Dove & Streptopelia chinensis & C \\
\hline 29. & Emerald Dove & Chalcophaps indica & $\mathrm{C}$ \\
\hline 30. & Rose-ringed Parakeet & Psittacula krameri & $\mathrm{C}$ \\
\hline 31. & Plum-headed Parakeet & Psittacula cyanocephala & $\mathrm{C}$ \\
\hline 32. & Vernal Hanging-Parrot & Loriculus vernalis & $\mathrm{C}$ \\
\hline 33. & Common Hawk Cuckoo & Cuculus varius & $\mathrm{F}$ \\
\hline 34. & Grey-bellied cuckoo (?) & Cacomantis passerinus & $\mathrm{R}$ \\
\hline 35. & Asian Koel & Eudynamys scolopacea & $\mathrm{C}$ \\
\hline 36. & Greater Coucal & Centropus sinensis & $\mathrm{C}$ \\
\hline 37. & Spotted Owlet & Athene brama & $\mathrm{C}$ \\
\hline 38. & Jungle Owlet & Glaucidium radiatum & $\mathrm{C}$ \\
\hline 39. & Collared Scops Owl & Otus bakkamoena & $\mathrm{F}$ \\
\hline 40. & Black-Nest Swiftlet & Collocalia fuciphaga & $\mathrm{F}$ \\
\hline 41. & Brown-backed Needletail Swift & Hirundapus giganteus & $\mathrm{R}$ \\
\hline 42. & White-rumped Needletail Swift & Zoonavena sylvatica & $\mathrm{R}$ \\
\hline 43. & Alpine Swift & Tachymarptis melba & $\mathrm{R}$ \\
\hline 44. & House Swift & Apus affinis & $\mathrm{C}$ \\
\hline 45. & Asian Palm Swift & Cypsiurus balasiensis & $\mathrm{C}$ \\
\hline 46. & Lesser Pied Kingfisher & Ceryle rudis & $\mathrm{R}$ \\
\hline 47. & Small Blue Kingfisher & Alcedo atthis & $\mathrm{F}$ \\
\hline 48. & White-breasted Kingfisher & Halcyon smyrnensis & $\mathrm{C}$ \\
\hline 49. & Stork-billed Kingfisher & Pelargopsis capensis & $\mathrm{R}$ \\
\hline 50. & Blue-tailed Bee-eater & Merops philippinus & $\mathrm{R}, \mathrm{M}$ \\
\hline 51. & Small Bee-eater & Merops orientalis & $\mathrm{F}$ \\
\hline 52. & Indian Roller & Coracias benghalensis & $\mathrm{F}$ \\
\hline 53. & Hoopoe & Upupa epops & $\mathrm{R}$ \\
\hline 54. & White-cheeked Barbet & Megalaima viridis & $\mathrm{C}$ \\
\hline 55. & Coppersmith Barbet & Megalaima haemacephala & $\mathrm{F}$ \\
\hline 56. & Rufous Woodpecker & Celeus brachyurus & $\mathrm{R}$ \\
\hline 57. & Lesser Golden-backed Woodpecker & Dinopium benghalense & $\mathrm{F}$ \\
\hline 58. & Common Golden-backed Woodpecker & Dinopium javanense & $\mathrm{R}$ \\
\hline 59. & Grey-capped Woodpecker & Dendrocopos canicapillus & $\mathrm{R}$ \\
\hline 60. & Indian Pitta & Pitta brachyura & $\mathrm{R}, \mathrm{M}$ \\
\hline 61. & Bengal bush-lark & Mirafra assamica & $\mathrm{C}$ \\
\hline 62. & Ashy Crowned Sparrow Lark & Eremopterix grisea & $\mathrm{R}$ \\
\hline 63. & Barn Swallow & Hirundo rustica & $\mathrm{R}$ \\
\hline 64. & Red-rumped Swallow & Hirundo fluvicola & $\mathrm{R}$ \\
\hline 65. & Brown Shrike & Lanius cristatus & $\mathrm{C}, \mathrm{M}$ \\
\hline 66. & Eurasian Golden Oriole & Oriolus oriolus & $\mathrm{F}, \mathrm{M}$ \\
\hline 67. & Black-hooded Oriole & Oriolus xanthornus & $\mathrm{C}$ \\
\hline 68. & Black Drongo & Dicrurus macrocercus & $\mathrm{C}$ \\
\hline 69. & Ashy Drongo & Dicrurus leucophaeus & $\mathrm{C}, \mathrm{M}$ \\
\hline 70. & Bronzed Drongo & Dicrurus aeneus & $\mathrm{F}$ \\
\hline 71. & Greater Racket-tailed Drongo & Dicrurus paradiseus & C \\
\hline 72. & Ashy Wood Swallow & Artamus fuscus & $\mathrm{C}$ \\
\hline 73. & Grey-headed Starling & Sturnus malabaricus & $\mathrm{C}, \mathrm{M}$ \\
\hline 74. & White-headed Starling & Sturnus erythropygius & $\mathrm{R}, \mathrm{LM}(?)$ \\
\hline
\end{tabular}




\begin{tabular}{|c|c|c|c|}
\hline S. No. & Common Name & Scientific Name & Status \\
\hline 75. & Common Myna & Acridotheres tristis & $\mathrm{C}$ \\
\hline 76. & Indian Treepie & Dendrocitta vagabunda & $\mathrm{C}$ \\
\hline 77. & House Crow & Corvus splendens & C \\
\hline 78. & Jungle Crow & Corvus macrorhynchos & $\mathrm{C}$ \\
\hline 79. & Black-headed Cuckoo-Shrike & Coracina melanoptera & $\mathrm{R}$ \\
\hline 80. & Large Cuckoo Shrike & Coracina macei & $\mathrm{R}$ \\
\hline 81. & Scarlet Minivet & Pericrocotus flammeus & C, LM (?) \\
\hline 82. & Small Minivet & Pericrocotus cinnamomeus & $\mathrm{F}$ \\
\hline 83. & Common lora & Aegithina tiphia & $\mathrm{F}$ \\
\hline 84. & Jerdon's Chloropsis & Chloropsis cochinchinensis & $\mathrm{F}$ \\
\hline 85. & Gold-fronted Chloropsis & Chloropsis aurifrons & $\mathrm{F}$ \\
\hline 86. & Red-whiskered Bulbul & Pycnonotus jocosus & C \\
\hline 87. & Red-vented Bulbul & Pycnonotus cafer & $\mathrm{C}$ \\
\hline 88. & White-browed Bulbul & Pycnonotus luteolus & $\mathrm{F}$ \\
\hline 89. & Rufous Babbler & Turdoides subrufus & $\mathrm{F}$ \\
\hline 90. & Jungle Babbler & Turdoides striatus & $\mathrm{F}$ \\
\hline 91. & Yellow-billed Babbler & Turdoides affinis & C \\
\hline 92. & Asian Brown Flycatcher & Muscicapa dauurica & $\mathrm{R}, \mathrm{M}$ \\
\hline 93. & Brown-breasted Flycatcher & Muscicapa muttui & $\mathrm{R}, \mathrm{M}$ \\
\hline 94. & Tickell's Blue-Flycatcher & Cyornis tickelliae & $\mathrm{R}$ \\
\hline 95. & Asian Paradise Flycatcher & Terpsiphone paradisi & $\mathrm{F}, \mathrm{M}$ \\
\hline 96. & Black-naped Monarch-Flycatcher & Hypothymis azurea & $\mathrm{F}$ \\
\hline 97. & Streaked Fantail-Warbler & Zisticola juncidis & $\mathrm{R}$ \\
\hline 98. & Plain Prinia & Prinia inornata & $\mathrm{R}$ \\
\hline 99. & Grey-breasted Prinia & Prinia hodgsonii & $\mathrm{R}$ \\
\hline 100. & Common Tailor Bird & Orthotomus sutorius & $\mathrm{C}$ \\
\hline 101. & Blyth's Reed Warbler & Acrocephalus dumetorum & $\mathrm{C}, \mathrm{M}$ \\
\hline 102. & Greenish Leaf-Warbler & Phylloscopus trochiloides & $\mathrm{C}, \mathrm{M}$ \\
\hline 103. & Oriental Magpie Robin & Copsychus saularis & $\mathrm{C}$ \\
\hline 104. & Indian Robin & Saxicoloides fulicatus & $\mathrm{C}$ \\
\hline 105. & Malabar Whistling Thrush & Myiophonus horsfieldii & $\mathrm{R}, \mathrm{LM}(?)$ \\
\hline 106. & Orange-headed (White-throated) Ground Thrush & Zoothera citrina & $\mathrm{F}, \mathrm{LM}(?)$ \\
\hline 107. & Eurasian Blackbird & Turdus merula & $\mathrm{F}, \mathrm{M}$ \\
\hline 108. & Paddy field Pipit & Anthus rufulus & C \\
\hline 109. & Grey Wagtail & Motacilla cinerea & $\mathrm{F}, \mathrm{M}$ \\
\hline 110. & White-browed Wagtail & Motacilla madaraspatensis & C \\
\hline 111. & Pale-billed Flowerpecker & Dicaeum erythrorhynchos & $\mathrm{F}$ \\
\hline 112. & Purple-rumped Sunbird & Nectarinia zeylonica & C \\
\hline 113. & Loten's Sunbird & Nectarinia lotenia & $\mathrm{C}$ \\
\hline 114. & House Sparrow & Passer domesticus & $\mathrm{F}$ \\
\hline 115. & Yellow-throated Sparrow & Petronia xanthocollis & $\mathrm{F}, \mathrm{M}$ \\
\hline 116. & Baya Weaver & Ploceus philippinus & $\mathrm{R}$ \\
\hline 117. & White-rumped Munia & Lonchura striata & C \\
\hline 118. & Spotted Munia & Lonchura punctulata & $\mathrm{R}$ \\
\hline
\end{tabular}

$\begin{array}{lll}\text { M } & \text { Migrant } & \text { Considered migratory in the whole of Kerala. } \\ \text { LM } & \text { Local Migrant } & \text { Migrating locally outside the campus } \\ \text { V } & \text { Vagrant } & \text { On passage. } \\ \text { C } & \text { Common } & \text { Indicates the species can be spotted during any normal outing (For migrants during the months they stay) } \\ \text { F } & \text { Fairly Common } & \text { Indicates the species can be spotted only after a few regular visits to the habitat. } \\ \text { R } & \text { Rare } & \text { Not more than five sightings during the study period. }\end{array}$

It should be realized that large college campuses like NIT has a role to play in conservation of natural eco-systems. These areas are possibly the least affected in terms of biotic pressure and hence can be used to harbor rich bio-diversity through careful planning.

\section{REFERENCES}

Ali, S. and S.D. Ripley (1986). Handbook of birds of India \& Pakistan together with those of Bangladesh, Nepal \& Bhutan,Vol 5, Oxford University Press, Delhi.

Grimmett, R., C. Inskipp and T. Inskipp (1999). A pocket guide to the birds of the Indian Subcontinent, $1^{\text {st }} \boldsymbol{E} d$, Oxford University Press, Delhi.

Palot, M.J. and P. Pramod (2000). A checklist of birds of Calicut University Campus. Zoos' Print Journal 15(2): 214-216.

Kazmierczak, K. (2000). A Field Guide to the Birds of the Indian Subcontinent, $1^{\text {st }}$ Ed, Pica Press, London. 